\title{
An Ultra-narrow-linewidth Microlaser for Nanosensing
}

\author{
Tao $\mathrm{Lu}^{1,2}$, Hansuek Lee ${ }^{1}$, Tong Chen ${ }^{1}$, Steven Herchak ${ }^{2}$ \\ 1. Applied Physics, California Institute of Technology, Pasadena, California, 91125 \\ 2. Electrical and Computer Engineering, University of Victoria, Victoria, BC, Canada
}

\begin{abstract}
We report the detection of 11.5-nm radius polystyrene beads in an aqueous environment at a signal-to-noise-ratio of 18:1 by monitoring the split frequency steps of a 5.7-KHz-linewidth microlaser. The thermal-optical effect is also observed.
\end{abstract}

OCIS codes: (230.3990) Micro-optical devices; (040.1880) Detection; (230.5750) Resonators.

\section{Introduction}

Microcavity-based nanosensing is a promising technology toward label-free, single protein molecule detection. To date, a record sensitivity in an aqueous environment using a silica microtoroid has been reported[1]. In that work, the toroid has an undoped cavity linewidth of $2 \mathrm{MHz}$. This leads to a minimum detectable frequency shift steps to the order of several hundred kilohertz. To enhance the sensitivity, an ultra-high quality factor (Q) based microlaser is desirable. As known, the laser fundamental linewidth is quadratic inverse proportional to the undoped cavity Q. Consequently, a high Q cavity significantly reduces the linewidth of a laser and enhances the frequency shift detection resolution. In addition, an ultra-high-Q laser displays a beat frequency in the microwave region due to mode-splitting effects[3]. The beat frequency linewidth is inherited from the laser fundamental linewidth while its center frequency changes upon a particle binding event. Unlike the change of resonance frequency at particle binding events, the change of beat frequency can be easily monitored with a spectrum analyzer and is immune to common mode noises such as those arise from temperature induced cavity optical path length fluctuations. This makes it an ideal tool for nanoparticle detection. Finally, due to the high pump power intensity at the cavity surface, the power absorption of the particle will thermally increase its refractive index, leading to a larger resonance and split frequency shift. In our work, a record split frequency linewidth as low as $5.7 \mathrm{KHz}$ is demonstrated. As a result, a record sensitivity on nanoparticle detection is reported.

\section{Experimental Results}

The schematic of the experiment setup is shown in Fig. 1(a). An Ytterbium (Yb) doped silica microtoroid $(\mathrm{Yb}$ concentration: $1 \times 10^{19} \mathrm{~cm}^{-3}$ is fabricated through a sol-gel process. The undoped cavity quality factor at the operating wavelength is estimated to be $5 \times 10^{6}$ from the optical absorption in the aqueous environment. A pump laser operating in the $970-980 \mathrm{~nm}$ wavelength range is coupled to the toroid through a tapered fiber, triggering a lasing action at $1030 \mathrm{~nm}$ wavelength. The laser output is coupled out through the same taper and separated into two branches by a directional coupler. One output arm of the coupler is connected to an optical spectrum analyzer for monitoring purposes while the laser output from the other arm is further separated from the residual pump light through a wavelength division multiplexer (WDM). Both outputs are converted to electrical signals by photo detectors with the signal of the pump connected to an oscilloscope for monitoring while that of the laser is acquired by an electrical spectrum analyzer. The nanoparticles diluted in Dulbecco's phosphate buffered saline (DPBS) are injected to the laser and the individual particle binding events are detected by locating the step-like shift of the beat frequency peak. A typical beat frequency spectrum of the laser is displayed in Fig. 1(b). As seen, a linewidth of $5.7 \mathrm{KHz}$ is obtained through a least square fit to the Lorentzian shape. This leads to an absolute detection sensitivty of peak frequency change down the same magnitude in the absence of noises. To quantify the background noise, a spectrogram was acquired and displayed in Fig. 1(c) when pure DPBS is injected. A standard deviation of 11 $\mathrm{KHz}$ is obtained in a time span of 40 milliseconds, limiting the detection of binding induced shift to such level. In our experiment, we measured a 100 pico-Molar, $11.5 \mathrm{~nm}$ polystyrene nano-beads diluted in DPBS. As displayed in Fig. 2(a), a step of $1.28 \pm 0.07 \mathrm{MHz}$ is observed at 77.0756 seconds with a signal-to-noise ratio of 18: 1. A smaller step of $0.51 \pm 0.07 \mathrm{MHz}$ is also detected at 77.0802 seconds on the same spectrogram. The increase of the measurement uncertainty arises from the background binding events on non-equatorial positions. A smaller background noise is achievable at lower concentration but at the price of a longer acquisition period. A collection of 510 steps from 479 spectrograms is displayed in Fig. 2(b) as a histogram. As seen, the maximum step size is larger than the theoretical predicted reactive shift of $0.25 \mathrm{MHz}$ (green box) but in agreement with the estimated 
total shift of $1.4 \mathrm{MHz}$ if we include the thermal optical induced frequency shift (assuming a $2 \mathrm{~mW}$ pump power). On the other hand, a $2.4 \pm 0.6 \mathrm{MHz}$ maximum step of $30 \mathrm{~nm}$ radius silica beads whose thermal optical effects are known to be negligible is observed from the corresponding histogram in Fig. 2(c). This is in a good agreement with the predicted reactive shift in the absence of thermal optical effect. The above observations suggest the existence of thermal optical effect.

\section{Conclusion}

In conclusion, we have demonstrated a laser operating in an aqueous environment with a split frequency linewidth of $5.7 \mathrm{KHz}$. With this laser, a detection of $11.5 \mathrm{~nm}$ radius polystyrene beads at a signal-to-noise exceeding 18:1 is achieved. Finally, a frequency shift enhancement suggesting the thermal optical effect is observed.

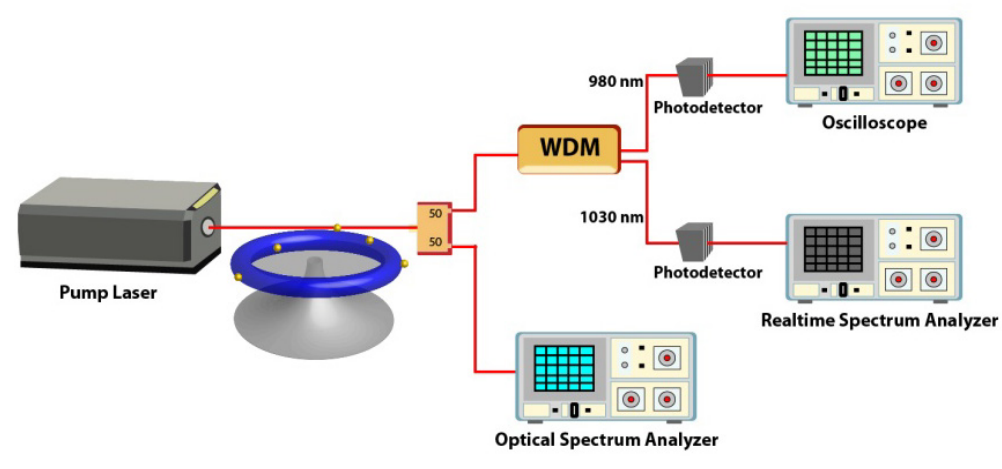

(a)
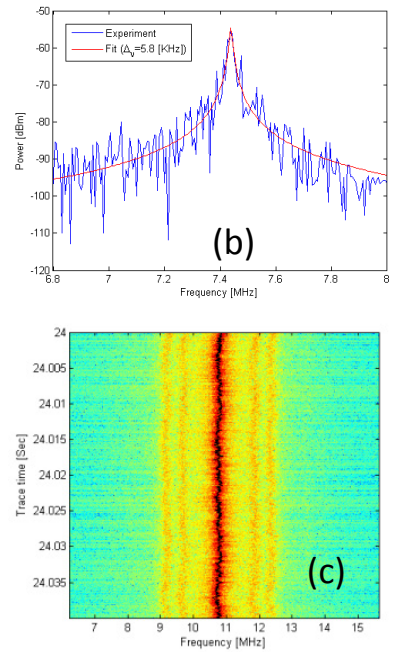

Fig. 1 (a) Experimental setup, (b) spectrum of the laser split frequency shows a linewidth of $5.7 \mathrm{KHz}$, (c) spectrogram of the split frequency measured in an aqueous environment displays a peak frequency standard deviation of $11 \mathrm{KHz}$, note the spectrogram is acquired from a different run than (b).
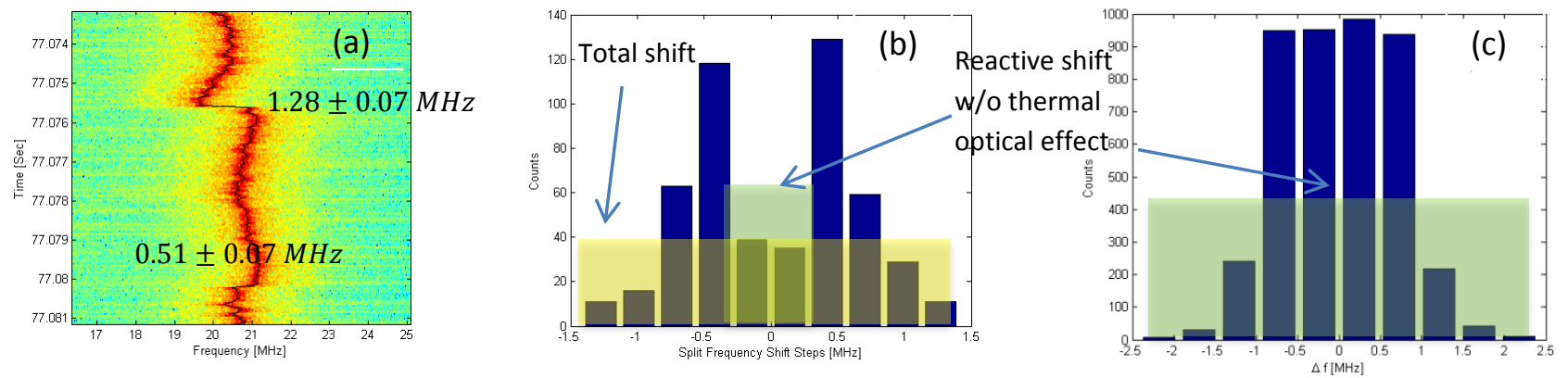

Fig. 2 (a) Spectrogram shows a beat frequency step of $1.28 \pm 0.07 \mathrm{MHz}$ at 77.0756 seconds and $0.51 \pm$ $0.07 \mathrm{M} \mathrm{Hz}$ at 77.0802 seconds. A histogram of a total 510 steps in (b) shows that the maximum step is larger than the theoretical prediction of the pure reactive shift (green box) but is in agreement with taking into consideration the thermal optical enhancement (yellow box). The histogram of $30 \mathrm{~nm}$ silica beads is shown in (c).

\section{References}

1. T. Lu, H. Lee, T. Chen, S. Herchak, J. Kim, S. Fraser, R. Flagan, and K. Vahala "High sensitivity nanoparticle detection using optical microcavities," PNAS 108 (15) 5976-5979(2011).

2. E. Ostby, L. Yang and K. J. Vahala, "Ultralow threshold $\mathrm{Yb}^{3+}: \mathrm{SiO}_{2}$ glass laser fabricated by the solgel process," Optics Letters, 32(18), 2650-2652(2007).

3. T. Lu, T.-T. J. Su, K. J. Vahala, and S. Fraser, "Split frequency sensing methods and systems," Patent pending, p. 20100085573, Oct. 2009, preliminary filing in Oct. 2008. 